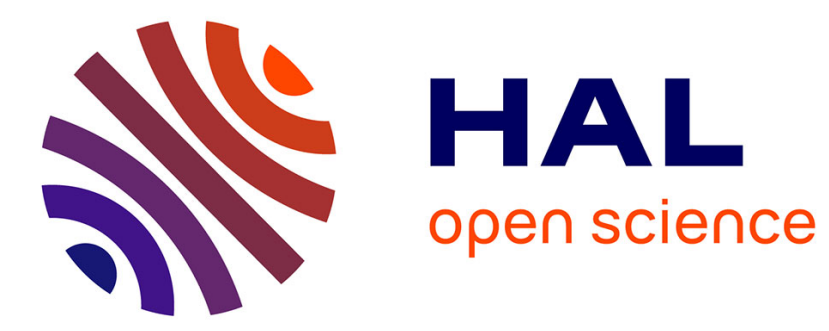

\title{
Convergence of a finite-volume scheme for a heat equation with a multiplicative stochastic force
}

\author{
Caroline Bauzet, Flore Nabet
}

\section{To cite this version:}

Caroline Bauzet, Flore Nabet. Convergence of a finite-volume scheme for a heat equation with a multiplicative stochastic force. Finite Volumes for Complex Applications IX, Jun 2020, Bergen, Norway. hal-02442422v2

HAL Id: hal-02442422

https://hal.science/hal-02442422v2

Submitted on 29 Jan 2020

HAL is a multi-disciplinary open access archive for the deposit and dissemination of scientific research documents, whether they are published or not. The documents may come from teaching and research institutions in France or abroad, or from public or private research centers.
L'archive ouverte pluridisciplinaire HAL, est destinée au dépôt et à la diffusion de documents scientifiques de niveau recherche, publiés ou non, émanant des établissements d'enseignement et de recherche français ou étrangers, des laboratoires publics ou privés. 


\title{
Convergence of a finite-volume scheme for a heat equation with a multiplicative stochastic force
}

\author{
Caroline Bauzet, Flore Nabet
}

\begin{abstract}
We present here the discretization by a finite-volume scheme of a heat equation perturbed by a multiplicative noise of Itô type and under homogeneous Neumann boundary conditions. The idea is to adapt well-known methods in the deterministic case for the approximation of parabolic problems to our stochastic PDE. In this paper, we try to highlight difficulties brought by the stochastic perturbation in the adaptation of these deterministic tools.
\end{abstract}

Key words: Stochastic heat equation, Itô integral, multiplicative noise, Itô formula, predictable process, Finite Volume Method.

MSC (2010): 35K05, 60H15, 65M08

\section{Introduction}

In this section, we present the stochastic heat equation we are studying. After deriving assumptions on the data, we explain the goal of the paper and give the definition of weak solution we are looking for. More precisely, we are interested in the following stochastic heat equation set in $(0, T) \times \Omega \times \Theta$,

$$
\partial_{t}\left(u(t, x, \omega)-\int_{0}^{t} \lambda u(s, x, \omega) d W(s)\right)-\Delta u(t, x, \omega)=0,
$$

Caroline Bauzet

Aix Marseille Univ, CNRS, Centrale Marseille, LMA

13013 Marseille, France

e-mail: caroline.bauzet@univ-amu.fr

Flore Nabet

CMAP, Ecole polytechnique, CNRS, I.P. Paris, 91128 Palaiseau, France

e-mail: flore.nabet@polytechnique.edu 
where $\Omega$ is an open bounded polygonal subset of $\mathbb{R}^{2}, T>0, W=\left\{W_{t}, \mathscr{F}_{t} ; 0 \leqslant t \leqslant\right.$ $T\}$ is a standard adapted one-dimensional continuous Brownian motion defined on the classical Wiener space $(\Theta, \mathscr{F}, \mathbb{P})$ and $\lambda \in \mathbb{R}$. An initial condition is given by a deterministic function $u_{0} \in L^{2}(\Omega)$ :

$$
u(0, x, \omega)=u_{0}(x), x \in \Omega, \omega \in \Theta,
$$

and we consider homogeneous Neumann boundary condition :

$$
\nabla u(t, x, \omega) \cdot \mathbf{n}(x)=0, t \in(0, T), x \in \partial \Omega, \omega \in \Theta,
$$

where $\mathbf{n}$ denotes the unit vector to $\partial \Omega$, outward to $\Omega$. In order to make the lecture more fluent, we omit in the sequel the random variable $\omega$. Note that the present study can be easily adapted to the case where $\Omega$ is a subset of $\mathbb{R}^{3}$ but for the sake of readability, we restrict the presentation to the 2 -dimensional case.

In this paper, the stochastic integral $\int_{0} \lambda u d W$ is understood in the sense of Itô, so that due to its non-anticipative construction with simple processes, we must consider an explicit time-discretization of this object. Note that the unknown $u$ appears in the stochastic integral, thus the noise is said to be multiplicative, otherwise it is additive.

The numerical analysis of heat equation (and generally second-order parabolic equations) under stochastic perturbation, random source term or random coefficients has been the subject of several studies by the way of finite-element approximations (see [7] for a thorough presentation of the state of the art on this subject). The aim of the present paper is to expose tools of stochastic calculus used to adapt known methods in the deterministic case to get the convergence of a suitable finite-volume scheme for the approximation of problem (1)-(2)-(3). This work stands for an introductive study in order to apprehend more complex problems such as the finitevolume approximation of stochastic nonlinear degenerate parabolic equations, having in mind the deterministic case treated by [6].

In what follows, we will show the convergence of a suitable numerical scheme through a stochastic process $u$, weak solution of (1)-(2)-(3) in the following sense:

Definition 1 A predictable process $u$ with values in $L^{2}(\Omega)$ is a weak solution of (1)(2)-(3) if $u \in L^{2}\left((0, T) \times \Theta ; H^{1}(\Omega)\right) \cap L^{\infty}\left((0, T) ; L^{2}(\Omega \times \Theta)\right)$ and if it satisfies $\mathbb{P}$ a.s in $\Theta$ and for any $\psi \in \mathscr{A}_{T}=\left\{\varphi \in C^{\infty}\left(\mathbb{R} \times \mathbb{R}^{2}\right): \varphi(T,)=0.\right\}$ the variational formulation

$$
\begin{aligned}
& \int_{0}^{T} \int_{\Omega} u(t, x) \partial_{t} \psi(t, x) d x d t-\int_{0}^{T} \int_{\Omega} \nabla u(t, x) \cdot \nabla \psi(t, x) d x d t+\int_{\Omega} u_{0}(x) \psi(0, x) d x \\
& =\int_{0}^{T} \int_{\Omega} \int_{0}^{t} \lambda u(s, x) d W(s) \partial_{t} \psi(t, x) d x d t
\end{aligned}
$$

Remark 1 The predictability property of $u$ with values in $L^{2}(\Omega)$ is a condition of measurability of the solution $u$ with respect to the filtration $\mathscr{F}=\left(\mathscr{F}_{t}\right)_{0 \leqslant t \leqslant T}$, which represents the history of the Brownian motion up to time $T$. It is required since we consider a multiplicative noise. More precisely, it means that $u$ belongs to $L^{2}\left((0, T) \times \Theta, \mathscr{P}_{T}, d t \otimes \mathbb{P} ; L^{2}(\Omega)\right)$ where $\mathscr{P}_{T}$ denotes the predictable $\sigma$-field gen- 
erated by (see $[8, \mathrm{p} .27])$

$$
\left\{X:(0, T) \times \Theta \rightarrow \mathbb{R}: X \text { is left-continuous and } \forall t \in[0, T], X_{t} \text { is } \mathscr{F}_{t} \text {-measurable }\right\} .
$$

We denote by $\mathscr{N}_{W}^{2}\left(0, T ; L^{2}(\Omega)\right)$ the space of predictable processes with values in $L^{2}(\Omega)$. Endowed with the norm $\|X\|^{2}=\int_{0}^{T} \int_{\Theta}\|X\|_{L^{2}(\Omega)}^{2} d \mathbb{P} d t$, it is a Hilbert space.

Remark 2 An application of Itô derivation formula (see [3, Theorem 4.17, p.105]) allows us to remark that the right-hand side of (4) can also be written in the following manner

$$
\int_{0}^{T} \int_{\Omega} \int_{0}^{t} \lambda u(s, x) d W(s) \partial_{t} \psi(t, x) d x d t=-\int_{\Omega} \int_{0}^{T} \lambda u(t, x) \psi(t, x) d W(t) d x .
$$

\section{Meshes, scheme and discrete norms}

We will use a classical two-point flux approximation scheme with an admissible mesh as in [5]. Firstly, we remind for convenience this definition adapted to our subset $\Omega$ of $\mathbb{R}^{2}$ and give some notations. Secondly, we present the finite-volume scheme used to approximate the weak solution $u$ of (1)-(2)-(3). Thirdly, we introduce discrete $L^{2}(\Omega)$-norm and $H^{1}(\Omega)$-seminorm used for the stability results exposed in the next section.

An admissible mesh $\mathscr{T}$ is given by a family of disjoint open polygonal subsets of $\Omega$, called "control volumes" and denoted by $K$ such that:

- $\bar{\Omega}=\cup_{K \in \mathscr{T}} \bar{K}$

- if $K, L \in \mathscr{T}, K \neq L$, then $\stackrel{\circ}{K} \cap \stackrel{\circ}{L}=\emptyset$;

- if $K, L \in \mathscr{T}, K \neq L$, either the 1-dimensional Lebesgue measure of $\bar{K} \cap \bar{L}$ is 0 or $\bar{K} \cap \bar{L}$ is the edge $\sigma$ of the mesh separating the control volumes $K$ and $L$;

- at each $K \in \mathscr{T}$, we associate a point $x_{K} \in K$, called the center of $K$, such that if $K, L$ are two neighbouring control volumes, the edge $\sigma=K \mid L$ which separates $K$ and $L$ is orthogonal to the straight line going through $x_{K}$ and $x_{L}$.

Once an admissible finite-volume mesh $\mathscr{T}$ of $\Omega$ is fixed, we will use in the sequel the following notations.

\section{Notations}

- $E[$.$] denotes the expectation, i.e the integral over \Theta$ with respect to the probability measure $\mathbb{P}$.

- $\mathscr{E}$ is the set of the edges of the mesh $\mathscr{T}$ and $\mathscr{E}$ int $=\{\sigma \in \mathscr{E}: \sigma \not \subset \partial \Omega\}$ the set of interior edges.

- For any $K \in \mathscr{T}, \mathscr{E}_{K}$ is the set of the edges of the control volume $K$ and $m_{K}$ the Lebesgue measure of $K$.

- For any $\sigma=K \mid L, m_{\sigma}$ is the length of $\sigma$ and $d_{K \mid L}$ the distance between the centers $x_{k}$ and $x_{L}$.

- $h=\operatorname{size}(\mathscr{T})=\sup \{\operatorname{diam}(K), K \in \mathscr{T}\}$, the mesh size. 
In order to compute an approximation of $u$ on $[0, T]$, we take $N \in \mathbb{N}^{*}$ and consider a fixed time step $\Delta t=\frac{T}{N} \in \mathbb{R}_{+}^{*}$. We first define the set $\left\{u_{K}^{0}, K \in \mathscr{T}\right\}$ by the discretization of the initial condition using its mean value over the control volume $K$,

$$
u_{K}^{0}=\frac{1}{m_{K}} \int_{K} u_{0}(x) d x .
$$

The equations satisfied by the discrete unknowns denoted by $u_{K}^{n}, n \in\{0, \cdots, N-1\}$, $K \in \mathscr{T}$ are given by the following explicit scheme

$$
\frac{m_{K}}{\Delta t}\left(u_{K}^{n+1}-u_{K}^{n}\right)+\sum_{\sigma \in \mathscr{E}_{K} \cap \mathscr{E}_{i n t}} \frac{m_{\sigma}}{d_{K \mid L}}\left(u_{K}^{n}-u_{L}^{n}\right)=\frac{m_{K}}{\Delta t} \lambda u_{K}^{n}\left(W^{n+1}-W^{n}\right),
$$

where $W^{n}=W(n \Delta t), \forall n \in\{0, \cdots, N\}$. Note that since the Brownian motion $W$ is standard, thus $W(0)=0$. The random dependence of the discrete unknowns $u_{K}^{n}$ $n \in\{1, \cdots, N\}$, comes from the randomness of the increments $W^{n+1}-W^{n}$, again for convenience, we omit the random variable $\omega$ and write $u_{K}^{n}$ instead of $u_{K}^{n}(\omega)$. We define the piecewise constant approximate solution $\left(u_{\mathscr{T}}^{\Delta t}\right)$ on $(0, T) \times \Omega \times \Theta$ from the discrete unknowns $u_{K}^{n}$ by

$$
u_{\mathscr{T}}^{\Delta t}(t, x, \omega)=u_{\mathscr{T}}^{n}(x, \omega)=u_{K}^{n}(\omega)=u_{K}^{n}, \quad t \in[n \Delta t,(n+1) \Delta t), x \in K, \omega \in \Theta,
$$

where $\left(u_{\mathscr{T}}^{n}\right)_{\mathscr{T}}$ defined on $\Omega \times \Theta$ is the sequence of the approximate solution at time $t^{n}=n \Delta t$ for $n \in\{0, \ldots, N\}$.

Remark 3 Let us mention that using properties of the Brownian motion $W$, for all $K \in \mathscr{T}$ and all $n \in\{0, \cdots, N\}, u_{K}^{n}$ is $\mathscr{F}_{n \Delta t}$-measurable. Thus, $u_{\mathscr{T}}^{\Delta t}$ is predictable with values in $L^{2}(\Omega)$ as an elementary process adapted to the filtration $\left(\mathscr{F}_{t}\right)_{0 \leqslant t \leqslant T}$.

We then define for any $n \in\{0, . ., N\}$ the following discrete $L^{2}(\Omega)$-norm $\|\cdot\|_{L^{2}(\Omega)}$ and $H^{1}(\Omega)$ seminorm $|\cdot|_{1, \mathscr{T}}^{2}$ for the approximate sequence $\left(u_{\mathscr{T}}^{n}\right)_{\mathscr{T}}, \mathbb{P}$-a.s in $\Theta$

$$
\left\|u_{\mathscr{T}}^{n}\right\|_{L^{2}(\Omega)}^{2}=\sum_{K \in \mathscr{T}} m_{K}\left|u_{K}^{n}\right|^{2} \text { and }\left|u_{\mathscr{T}}^{n}\right|_{1, \mathscr{T}}^{2}=\sum_{\sigma=K \mid L \in \mathscr{E}_{i n t}} \frac{m_{\sigma}}{d_{K \mid L}}\left|u_{K}^{n}-u_{L}^{n}\right|^{2} .
$$

\section{Convergence of the scheme}

In this section, we propose a study of the approximate sequence $\left(u_{\mathscr{T}}^{\Delta t}\right)$. After the derivation of boundedness estimates for $\left(u_{\mathscr{T}}^{\Delta t}\right)$ independent of the discretization parameters $\Delta t$ and $h$, we propose to show the convergence of $\left(u_{\mathscr{T}}^{\Delta t}\right)$ towards a weak solution $u$ of (1)-(2)-(3) in the sense of Definition 1. 
Proposition 1 Let $T>0, \mathscr{T}$ be an admissible mesh, $N \in \mathbb{N}^{*}$ and $\Delta t=\frac{T}{N} \in \mathbb{R}_{+}^{*}$. Assume that the condition

$$
\frac{\Delta t}{m_{K}} \sum_{\sigma \in \mathscr{E}_{K} \cap \mathscr{E}_{i n t}} \frac{m_{\sigma}}{d_{K \mid L}} \leqslant \frac{1}{2}, \forall K \in \mathscr{T}
$$

is satisfied. Then there exists a constant $C>0$ only depending on $T, \Omega, \lambda$ and $u_{0}$ such that

$$
\sup _{n \leqslant N} E\left[\left\|u_{\mathscr{T}}^{n}\right\|_{L^{2}(\Omega)}^{2}\right]+\sum_{n=0}^{N-1} \Delta t E\left[\left|u_{\mathscr{T}}^{n}\right|_{1, \mathscr{T}}^{2}\right] \leqslant C .
$$

Proof We will principally use here properties of the Brownian motion $W$ for the control of its discrete increments $W^{n+1}-W^{n}$ (see [3, p.87]). For any $n \in\{0, \cdots, N-$ $1\}$, note that since $E\left[W^{n+1}-W^{n}\right]=0$, one gets that for any $\mathscr{F}_{n \Delta t}$-measurable random variable $X: \Theta \rightarrow \mathbb{R}, E\left[\left(W^{n+1}-W^{n}\right) X\right]=E\left[W^{n+1}-W^{n}\right] E[X]=0$. Thus, by multiplying (6) by $u_{K}^{n}$ and taking the expectation, since $a(b-a)=\frac{1}{2}\left(b^{2}-a^{2}-(a-\right.$ $b)^{2}$ ) for any $a, b \in \mathbb{R}$, one gets

$$
\frac{m_{K}}{2 \Delta t} E\left[\left|u_{K}^{n+1}\right|^{2}-\left|u_{K}^{n}\right|^{2}-\left|u_{K}^{n+1}-u_{K}^{n}\right|^{2}\right]+\sum_{\sigma \in \mathscr{E}_{K} \cap \mathscr{E}_{i n t}} \frac{m_{\sigma}}{d_{K \mid L}} E\left[\left(u_{K}^{n}-u_{L}^{n}\right) u_{K}^{n}\right]=0 .
$$

Moreover thanks to (6), by noting that $E\left[\left(W^{n+1}-W^{n}\right)^{2}\right]=\Delta t$, one has

$$
E\left[\left|u_{K}^{n+1}-u_{K}^{n}\right|^{2}\right]=\Delta t E\left[\left|\lambda u_{K}^{n}\right|^{2}\right]+E\left[\left(\frac{\Delta t}{m_{K}} \sum_{\sigma \in \mathscr{E}_{K} \cap \mathscr{E}_{i n t}} \frac{m_{\sigma}}{d_{K \mid L}}\left(u_{K}^{n}-u_{L}^{n}\right)\right)^{2}\right],
$$

and one arrives at

$$
\begin{aligned}
& \frac{m_{K}}{2} E\left[\left|u_{K}^{n+1}\right|^{2}-\left|u_{K}^{n}\right|^{2}\right]+\Delta t \sum_{\sigma \in \mathscr{E}_{K} \cap \mathscr{E}_{i n t}} \frac{m_{\sigma}}{d_{K \mid L}} E\left[\left(u_{K}^{n}-u_{L}^{n}\right) u_{K}^{n}\right] \\
\leqslant & \Delta t \frac{m_{K}}{2} \lambda^{2} E\left[\left|u_{K}^{n}\right|^{2}\right]+\frac{\Delta t}{2}\left(\frac{\Delta t}{m_{K}} \sum_{\sigma \in \mathscr{E}_{K} \cap \mathscr{E}_{i n t}} \frac{m_{\sigma}}{d_{K \mid L}}\right)\left(\sum_{\sigma \in \mathscr{E}_{K} \cap \mathscr{E}_{i n t}} \frac{m_{\sigma}}{d_{K \mid L}} E\left[\left|u_{K}^{n}-u_{L}^{n}\right|^{2}\right]\right) .
\end{aligned}
$$

Summing over $K \in \mathscr{T}$ and using the condition (8), thanks to classical reorderings of the summations, we obtain

$$
\begin{aligned}
& \sum_{K \in \mathscr{T}} m_{K} E\left[\left|u_{K}^{n+1}\right|^{2}\right]+2 \Delta t \sum_{\sigma=K \mid L \in \mathscr{E}_{i n t}} \frac{m_{\sigma}}{d_{K \mid L}} E\left[\left|u_{K}^{n}-u_{L}^{n}\right|^{2}\right] \\
& \leqslant\left(1+\Delta t \lambda^{2}\right) \sum_{K \in \mathscr{T}} m_{K} E\left[\left|u_{K}^{n}\right|^{2}\right]+\frac{1}{2} \Delta t \sum_{K \in \mathscr{T}} \sum_{\sigma \in \mathscr{E}_{K} \cap \mathscr{E}_{i n t}} \frac{m_{\sigma}}{d_{K \mid L}} E\left[\left|u_{K}^{n}-u_{L}^{n}\right|^{2}\right]
\end{aligned}
$$

which leads to

$$
E\left[\left\|u_{\mathscr{T}}^{n+1}\right\|_{L^{2}(\Omega)}^{2}\right]+\Delta t E\left[\left|u_{\mathscr{T}}^{n}\right|_{1, \mathscr{T}}^{2}\right] \leqslant\left(1+\Delta t \lambda^{2}\right) E\left[\left\|u_{\mathscr{T}}^{n}\right\|_{L^{2}(\Omega)}^{2}\right] .
$$


Summing (9) over $n \in\{0, \cdots, m\}$, the discrete Gronwall lemma gives the expected $L^{\infty}\left(0, T ; L^{2}(\Omega \times \Theta)\right)$ bound,

$$
E\left[\left\|u_{\mathscr{T}}^{m}\right\|_{L^{2}(\Omega)}^{2}\right] \leqslant\left(1+\Delta t \lambda^{2}\right) e^{\lambda^{2} T}\left\|u_{0}\right\|_{L^{2}(\Omega)}^{2}, \quad \forall m \in 0, \cdots, N .
$$

Summing (9) over $n \in\{0, \cdots, N-1\}$ we obtain the $L^{2}\left((0, T) \times \Theta ; H^{1}(\Omega)\right)$ bound,

$$
\sum_{n=0}^{N-1} \Delta t E\left[\left|u_{\mathscr{T}}^{n}\right|_{1, \mathscr{T}}^{2}\right] \leqslant\left(1+T \lambda^{2}\left(1+\Delta t \lambda^{2}\right) e^{\lambda^{2} T}\right)\left\|u_{0}\right\|_{L^{2}(\Omega)}^{2} .
$$

Remark 4 Let us mention that the stochastic perturbation does not impact the condition (8) on the time and space discretization parameter to get a stability result on the finite-volume approximation $\left(u_{\mathscr{T}}^{\Delta t}\right)$. Indeed, the condition is the same as in the deterministic case (which corresponds to $\lambda=0$ ).

Remark 5 Note that Proposition 1 also holds for a more general stochastic noise taking the form $\int_{0} g(u) d W$ with $g: \mathbb{R} \rightarrow \mathbb{R}$ Lipschitz-continuous. It also implies boundedness of the sequence $\left(g\left(u_{\mathscr{T}}^{\Delta t}\right)\right)$ and so the existence of a weak limit $g_{u}$ in $\mathscr{N}_{W}^{2}\left((0, T) ; L^{2}(\Omega)\right)$ for a subsequence of $\left(g\left(u_{\mathscr{T}}^{\Delta t}\right)\right)$. When $g$ is not a linear function, the convergence result stated in Theorem 1 below requires a compactness tool compatible with the random variable in order to affirm that $g_{u}=g(u)$. This extension will be carried out in a future work.

Theorem 1 For $m \in \mathbb{N}$, let $\mathscr{T}_{m}$ be an admissible mesh, $N_{m} \in \mathbb{N}^{*}$ and $\Delta t_{m}=\frac{T}{N_{m}}$ satisfying the condition (8). Let $\left(u_{\mathscr{T}_{m}}^{\Delta t_{m}}\right)$ be given by (5)-(6)-(7) with $\mathscr{T}=\mathscr{T}_{m}$ and $N=$ $N_{m}$. Then there exists a subsequence of $\left(u_{\mathscr{T}_{m}}^{\Delta t_{m}}\right)$, still denoted $\left(u_{\mathscr{T}_{m}}^{\Delta t_{m}}\right)$, which converges weakly in $L^{2}((0, T) \times \Omega \times \Theta)$ towards a weak solution $u$ of (1)-(2)-(3) in the sense of Definition 1 .

Proof We will only give here the idea of the proof to handle the stochastic term and refer to [5, Proof of Theorem 18.1 p.858] for the deterministic contributions. Let $m \in \mathbb{N}, A$ be a $\mathbb{P}$-measurable set and $\psi \in C^{\infty}\left(\mathbb{R} \times \mathbb{R}^{2}\right)$ such that $\psi(T,)=$.0 and $\nabla \psi \cdot \mathbf{n}=0$ on $(0, T) \times \partial \Omega$. Since $\Omega$ is a polygonal subset of $\mathbb{R}^{2}$, the set of such functions $\psi$ is dense in the set $\mathscr{A}_{T}$ for the $L^{2}\left(0, T ; H^{1}(\Omega)\right)$-norm (see [4]). For the sake of simplicity we shall use the notations $\mathscr{T}=\mathscr{T}_{m}, h=\operatorname{size}\left(\mathscr{T}_{m}\right)$ and $\Delta t=\Delta t_{m}$. We define the piecewise constant in space function $\psi_{\mathscr{T}}$ on $(0, T) \times \Omega$ by

$$
\psi_{\mathscr{T}}(t, x)=\frac{1}{m_{B_{K}}} \int_{B_{K}} \psi(t, y) d y, \quad \forall x \in K, t \in(0, T),
$$

where $B_{K} \subset K$ is a ball centered at $x_{K}$ and $m_{B_{K}}$ denotes its Lebesgue measure. We multiply (6) by $\Delta t 1_{A} \psi_{\mathscr{T}}\left(n \Delta t, x_{K}\right)$, sum the result over $n \in\{0, \cdots, N-1\}$ and $K \in$ $\mathscr{T}$, to get after taking the expectation : $T_{1, m}+T_{2, m}=T_{3, m}$, where 


$$
\begin{aligned}
& T_{1, m}=E\left[1_{A} \sum_{n=0}^{N-1} \sum_{K \in \mathscr{T}} m_{K}\left(u_{K}^{n+1}-u_{K}^{n}\right) \frac{1}{m_{B_{K}}} \int_{B_{K}} \psi(n \Delta t, x) d x\right] \\
& T_{2, m}=E\left[1_{A} \sum_{n=0}^{N-1} \Delta t \sum_{K \in \mathscr{T}} \sum_{\sigma \in \mathscr{E}_{K} \cap \mathscr{E}_{i n t}} \frac{m_{\sigma}}{d_{K \mid L}} \times \frac{\left(u_{K}^{n}-u_{L}^{n}\right)}{m_{B_{K}}} \int_{B_{K}} \psi(n \Delta t, x) d x\right] \\
& T_{3, m}=E\left[1_{A} \sum_{n=0}^{N-1} \sum_{K \in \mathscr{T}} \lambda m_{K} u_{K}^{n}\left(W^{n+1}-W^{n}\right) \frac{1}{m_{B_{K}}} \int_{B_{K}} \psi(n \Delta t, x) d x\right] .
\end{aligned}
$$

Proposition 1 allows us to extract firstly a subsequence of $\left(u_{\mathscr{T}}^{\Delta t}\right)$, still denoted $\left(u_{\mathscr{T}}^{\Delta t}\right)$, which converges as $m \rightarrow+\infty$ to an element $u$ in $L^{\infty}\left((0, T) ; L^{2}(\Omega \times \Theta)\right)$ for the weak- $\star$ topology. Secondly, since $\left(u_{\mathscr{T}}^{\Delta t}\right)$ is bounded in $\mathscr{N}_{W}^{2}\left(0, T ; L^{2}(\Omega)\right)$, one can affirm that $u$ is predictable with values in $L^{2}(\Omega)$ (see Remark 1) and that the previous convergence also holds (up to a subsequence) for the weak topology in $\mathscr{N}_{W}^{2}\left(0, T ; L^{2}(\Omega)\right)$. Now, following [1] (see Remark 6), [2] and [5], one shows that

$$
\begin{aligned}
& T_{1, m} \underset{m \rightarrow+\infty}{\longrightarrow}-E\left[1_{A} \int_{0}^{T} \int_{\Omega} u(t, x) \partial_{t} \psi(t, x) d x d t\right]-E\left[1_{A} \int_{\Omega} u_{0}(x) \psi(x, 0) d x\right] \\
& T_{2, m} \underset{m \rightarrow+\infty}{\longrightarrow}-E\left[1_{A} \int_{0}^{T} \int_{\Omega} u(t, x) \Delta \psi(t, x) d x d t\right] .
\end{aligned}
$$

By adapting the classical result of the two-point flux approximation scheme, one shows that $u \in L^{2}\left((0, T) \times \Theta ; H^{1}(\Omega)\right)$ by introducing a definition of discrete gradient for $\left(u_{\mathscr{T}}^{\Delta t}\right)$. Since the stochastic integral $I_{T}: X \mapsto \int_{0}^{T} X(t, x, \omega) d W(t)$ is linear and continuous from $\mathscr{N}_{W}^{2}\left(0, T ; L^{2}(\Omega)\right)$ to $L^{2}(\Omega \times \Theta)$, it is particularly weakly continuous and so the regularity of $\psi$ gives

$E\left[1_{A} \int_{\Omega} \int_{0}^{T} u_{\mathscr{T}}^{\Delta t}(t, x) \psi_{\mathscr{T}}(t, x) d W(t) d x\right] \underset{m \rightarrow+\infty}{\longrightarrow} E\left[1_{A} \int_{\Omega} \int_{0}^{T} u(t, x) \psi(t, x) d W(t) d x\right]$.

Using successively Cauchy-Schwarz inequality on $\Omega \times \Theta$, Itô isometry and Proposition 1, one shows by following [2] in the hyperbolic setting that (using the notation $|\Omega|$ for the area of $\Omega$ )

$$
\begin{aligned}
& \left|T_{3, m}-E\left[1_{A} \int_{\Omega} \int_{0}^{T} \lambda u_{\mathscr{T}}^{\Delta t}(t, x) \psi_{\mathscr{T}}(t, x) d W(t) d x\right]\right| \\
\leqslant & \sqrt{|\Omega|} \sum_{n=0}^{N-1}\left(\sum_{K \in \mathscr{T}} E\left[\int_{K}\left|\int_{n \Delta t}^{(n+1) \Delta t} \lambda u_{K}^{n}\left(\psi_{\mathscr{T}}(n \Delta t, x)-\psi_{\mathscr{T}}(t, x)\right) d W(t)\right| d x\right]\right)^{2} \\
= & \sqrt{|\Omega|} \sum_{n=0}^{N-1}\left(\sum_{K \in \mathscr{T}} E\left[\int_{n \Delta t}^{(n+1) \Delta t} \int_{K}\left|\lambda u_{K}^{n}\left(\psi_{\mathscr{T}}(n \Delta t, x)-\psi_{\mathscr{T}}(t, x)\right)\right|^{2} d x d t\right]\right)^{\frac{1}{2}} \\
\leqslant & \lambda|T \sqrt{|\Omega|}| \mid \partial_{t} \psi \|_{\infty} \sup _{n \leqslant N} E\left[\left\|u_{\mathscr{T}}^{n}\right\|_{L^{2}(\Omega)}^{2}\right] \sqrt{\Delta t} \underset{m \rightarrow+\infty}{\longrightarrow} 0 .
\end{aligned}
$$


Thus $T_{3, m} \underset{m \rightarrow+\infty}{\longrightarrow} E\left[1_{A} \int_{\Omega} \int_{0}^{T} \lambda u(t, x) \psi(t, x) d W(t) d x\right]$. Finally, for any $\psi \in \mathscr{A}_{T}$ and any $\mathbb{P}$-measurable set $A$, one gets

$$
\begin{aligned}
E & {\left[1_{A} \int_{0}^{T} \int_{\Omega} u(t, x) \partial_{t} \psi(t, x) d x d t\right]+E\left[1_{A} \int_{\Omega} u_{0}(x) \psi(0, x) d x\right] } \\
-E & {\left[1_{A} \int_{0}^{T} \int_{\Omega} \nabla u(t, x) \cdot \nabla \psi(t, x) d x d t\right]=-E\left[1_{A} \int_{\Omega} \int_{0}^{T} \lambda u(t, x) \psi(t, x) d W(t) d x\right] }
\end{aligned}
$$

and the result holds using Remark 2.

Remark 6 In the deterministic case, Theorem 1 is classically proved by multiplying (6) by $\Delta t \psi\left(n \Delta t, x_{K}\right)$ where $x_{K}$ is the center of the control volume $K$. Here, the application of Itô isometry gives us a coefficient $\sqrt{\Delta t}$ which is not sufficient to compensate the summation over $n \in\{0, \cdots, N-1\}$. Indeed, in this case there exists $\tilde{C}_{\psi}>0$ which only depends on $\psi$ such that

$$
\begin{aligned}
\sqrt{|\Omega|} \sum_{n=0}^{N-1}\left(\sum_{K \in \mathscr{T}} E\right. & {\left.\left[\int_{n \Delta t}^{(n+1) \Delta t} \int_{K}\left|\lambda u_{K}^{n}\left(\psi\left(n \Delta t, x_{K}\right)-\psi(t, x)\right)\right|^{2} d x d t\right]\right)^{\frac{1}{2}} } \\
& \leqslant \sqrt{\Delta t} \sqrt{|\Omega|}|\lambda|\left(\sup _{n \leqslant N} E\left[\|\left. u_{\mathscr{T}}^{n}\right|_{L^{2}(\Omega)} ^{2}\right]\right)^{\frac{1}{2}} \tilde{C}_{\psi} \sum_{n=0}^{N-1}(\Delta t+h),
\end{aligned}
$$

but this last term does not converge to 0 when $m$ tends to $+\infty$ under reasonable assumptions over $h$ and $\Delta t$. Choosing here the mean-value on $B_{K}$ allows us to show both the convergence of the terms $T_{3, m}$ and $T_{2, m}$ (using for $T_{2, m}$ similar arguments as in the deterministic framework, see [1, Proposition 3.5]).

\section{References}

1. Andreianov, B., Boyer, F., Hubert, F.: Discrete duality finite volume schemes for Leray-Lionstype elliptic problems on general 2D meshes. Numer. Methods Partial Differential Equations 23(1), 145-195 (2007)

2. Bauzet, C., Charrier, J., Gallouët, T.: Convergence of monotone finite volume schemes for hyperbolic scalar conservation laws with multiplicative noise. Stochastic Partial Differential Equations : Analysis and Computations 4, 150-223 (2016)

3. Da Prato, G., Zabczyk, J.: Stochastic equations in infinite dimensions, Encyclopedia of Mathematics and its Applications, vol. 44. Cambridge University Press, Cambridge (1992)

4. Droniou, J.: A density result in Sobolev spaces. J. Math. Pures Appl. (9) 81(7), 697-714 (2002)

5. Eymard, R., Gallouët, T., Herbin, R.: Finite volume methods. In: Handbook of numerical analysis, Vol. VII, Handb. Numer. Anal., VII, pp. 713-1020. North-Holland, Amsterdam (2000)

6. Eymard, R., Gallouët, T., Herbin, R., Michel, A.: Convergence of a finite volume scheme for nonlinear degenerate parabolic equations. Numer. Math. 92(1), 41-82 (2002)

7. Martel, S.: Theoretical and numerical analysis of invariant measures of viscous stochastic scalar conservation laws. Ph.D. thesis, Université Paris-Est (2019)

8. Prévôt, C., Röckner, M.: A concise course on stochastic partial differential equations, Lecture Notes in Mathematics, vol. 1905. Springer, Berlin (2007) 